\title{
Baja prevalencia de obesidad y alta prevalencia de bajo peso en pobladores de los Andes de Venezuela. Resultados preliminares del Estudio EVESCAM
}

\author{
María M. Infante-García ${ }^{1}$, Juan P. González-Rivas ${ }^{1}$, José Valencia Portillo ${ }^{2}$, Mariflor Vera \\ Eunice Ugel ${ }^{4}$, Maritæa Durán ${ }^{5}$, María Inés Marulanda ${ }^{6,7}$ y Ramfis Nieto-Martínes ${ }^{8,9}$ \\ ${ }^{1}$ Clínica de Estudios Cardiometabólicos los Andes, Mérida, Venezuela. \\ ${ }^{2}$ Escuela de Medicina, Universidad de los Andes (ULA), Mérida, Venezuela. \\ ${ }^{3}$ Departamento de Laboratorio Multidisciplinario de Investigación Clínico- \\ epidemiológica (Lab-MICE), Escuela de Medicina, Universidad de los Andes (ULA), \\ Mérida, Venezuela. \\ ${ }^{4}$ Unidad de Investigación de Salud Pública, Departamento de Medicina Preventiva \\ y Social, Escuela de Medicina, Universidad Centro-Occidental "Lisandro Alvarado", \\ Barquisimeto, Venezuela. \\ ${ }^{5}$ Departamento de Medicina Interna, Clínica Ávila, Caracas, Venezuela. \\ ${ }^{6}$ Endocrinos Asociados de Florida, Departamento de Investigación, Orlando, Florida, USA. \\ ${ }^{7}$ Departamento de Medicina Interna, Universidad de Carabobo y Centro Médico "Guerra \\ Mendez", Valencia, Venezuela. \\ ${ }^{8}$ Departamento de Fisiología, Escuela de Medicina, Universidad Centro-Occidental \\ "Lisandro Alvarado" y Unidad Cardiometabólica 7, Barquisimeto, Venezuela. \\ ${ }^{9}$ Geriatric Research, Education, and Clinical Center (GRECG) and South Florida \\ Veterans Affairs Foundation for Research \& Education, Miami VA Healtheare System, \\ Miami, FL, USA.
}

Palabras clave: obesidad; sobrepeso; bajo peso; IMC; grasa corporal; obesidad abdominal.

Resumen. La última evaluación del estado nutricional de la población que incluyó la región de Los Andes en Venezuela fue en el período 2006 a 2010, con resultados de 29,3\% de prevalencia de obesidad y $1,3 \%$ de bajo peso. El objetivo del presente estudio fue determinar el estado nutricional en una muestra poblacional de la región de los Andes evaluada en el estudio EVESCAM. Durante 2015 y 2016, fueron seleccionados 418 sujetos de 20 o más años de edad mediante un muestreo aleatorio estratificado por etapas de 5 poblaciones de la región de los Andes venezolanos. La tasa de respuesta fue 76,6\% (418 sujetos evaluados de 546 reclutados). El estado nutricional se definió acorde al índice

Autor de Correspondencia: María M. Infante-García; Mérida, Avenida Las Américas. Clínica de Estudios Cardiometabólicos los Andes. Mérida, Venezuela. Teléfono: +58424-7596482. Correo electrónico: mariainfante384@gmail.com. 
de masa corporal (IMC): bajo peso $<18,5 \mathrm{~kg} / \mathrm{m}^{2}$; peso normal 18,5 a $24,9 \mathrm{~kg}$ / $\mathrm{m}^{2}$; sobrepeso 25 a $29,9 \mathrm{~kg} / \mathrm{m}^{2}$; obesidad $\geq 30 \mathrm{~kg} / \mathrm{m}^{2}$. La obesidad abdominal se definió como circunferencia abdominal $\geq 94 \mathrm{~cm}$ en hombres y $\geq 90 \mathrm{~cm}$ en mujeres. Alto porcentaje de grasa se definió como $>25 \%$ en los hombres y $>35 \%$ en las mujeres. La prevalencia de obesidad fue $22,2 \%$, sobrepeso $39,9 \%$ y bajo peso $2,4 \%$. Las mujeres presentaron una mayor prevalencia de obesidad y bajo peso que los hombres $(\mathrm{p}=0,014)$. La prevalencia de bajo peso fue más elevada en Tovar con $8,8 \%(\mathrm{p}<0,05)$. Obesidad abdominal y alto porcentaje de grasa se presentaron en la mitad de la población. En conclusión, una baja prevalencia de obesidad y alta prevalencia de bajo peso fue observada en la población evaluada.

\title{
Low prevalence of obesity and high prevalence of underweight in the population of the Andes Region of Venezuela. Preliminary results of the EVESCAM Study
}

Invest Clin 2018; 59 (3): 242 - 249

Key words: obesity; overweight; underweight; BMI; body fat; abdominal obesity.

\begin{abstract}
The last evaluation of the nutritional status that included the Andes region was from 2006 to 2010 , the results were $29.3 \%$ prevalence of obesity and $1.3 \%$ of underweight. The aim of the study was to determine the nutritional status from a representative sample in the Andes region assessed in the EVESCAM study. From 2015-2016, 418 subjects aged $\geq 20$ years were selected by a multistage stratified random sampling in five populations from the Andes region. The response rate was $76.6 \%$, (418 subjects evaluated from 546 recruited). The nutritional status was defined according to the body mass index (BMI): underweight $<18.5 \mathrm{~kg} / \mathrm{m}^{2}$; normal weight 18.5 to $24.9 \mathrm{~kg} / \mathrm{m}^{2}$; overweight 25 to $29.9 \mathrm{~kg} / \mathrm{m}^{2}$; and obesity $\geq 30 \mathrm{~kg} / \mathrm{m}^{2}$. Abdominal obesity was defined as waist circumference $\geq 94 \mathrm{~cm}$ in men and $\geq 90 \mathrm{~cm}$ in women. High fat percent was defined as $>25 \%$ in men and $>35 \%$ in women. The prevalence of obesity was $22.2 \%$, overweight $39.9 \%$, and underweight $2.4 \%$. Women presented a higher prevalence of obesity and underweight than men did $(p=0,014)$. Half of the sample presented abdominal obesity and high body fat percent. The highest prevalence of underweight was in Tovar (Mérida) with $8.8 \%(\mathrm{p}<0.05)$. In conclusion, a low prevalence of obesity and a high prevalence of underweight was observed in the sample evaluated.
\end{abstract}

Recibido: 22-02-2018 Aceptado: 04-07-2018

Vol. 59(3): $242-249,2018$ 


\section{INTRODUCCIÓN}

El índice de masa corporal (IMC) aumentado representó globalmente el cuarto mayor factor de riesgo con 120,1 millones de años de vida perdidos por incapacidad o muertes para 2015, detrás de la presión arterial elevada, consumo de cigarrillos y glucosa en ayunas elevada (1). El IMC elevado incrementa el riesgo de hipertensión arterial, diabetes, depresión, osteoartritis, ciertos tipos de cáncer y apnea del sueño $(2,3)$.

La prevalencia de obesidad (IMC $\geq 30$ $\mathrm{kg} / \mathrm{m}^{2}$ ), estandarizada por edad, se incrementó ǵlobalmente de $3,2 \%$ a $10,8 \%$ en hombres $\mathrm{y}$ de $6,4 \%$ a $14,9 \%$ en mujeres desde 1975 a 2014 (4). En este mismo periodo, la prevalencia de bajo peso decreció de 13,8\% a 8,8\% en hombres y de $14,6 \%$ a $9,7 \%$ en mujeres (4). En Venezuela no se había realizado una encuesta nacional para determinar la prevalencia de sobrepeso y obesidad. Sin embargo, basados en estudios regionales y sub-nacionales publicados, que evaluaron 3.368 sujetos, la prevalencia estimada de obesidad (IMC $\geq$ $30 \mathrm{~kg} / \mathrm{m}^{2}$ ) ponderada en los participantes de los estudios en Venezuela, fue 26,3\% entre 2006 a 2010.(5) El estudio más representativo desarrollado en Venezuela fue el estudio Venezolano de Síndrome Metabólico, Obesidad y Estilo de Vida (VEMSOLS), que evaluó 1.320 adultos entre los años 2006 a 2010 en 3 regiones del país (Andes, Occidente y Capital) y reportó una prevalencia de obesidad en la población de los Andes de $24,3 \%$ en la zona urbana y de $12,1 \%$ en la zona rural del Páramo $(6,7)$. La prevalencia de bajo peso en las tres regiones fue de $1,1 \%$ para $2010(7)$. En vista que desde 2010 no se ha desarrollado ningún estudio que reporte de forma representativa la prevalencia del estado nutricional de los sujetos de la región de los Andes, se planteó realizar el presente estudio con el objetivo de determinar el estado nutricional en una muestra de esta región evaluada en el estudio Estudio Venezolano de Salud CardioMetabólica (EVESCAM) y analizar los resultados entre géneros y localidades estudiadas.

\section{MATERIAL Y MÉTODOS}

\section{Diseño y Población}

El diseño, muestreo e implementación del estudio, han sido descritos previamente $(8,9)$. Brevemente, el EVESCAM fue un estudio poblacional, observacional, transversal $\mathrm{y}$ de muestreo aleatorio por etapas y por conglomerados, diseñado para evaluar la salud cardiometabólica de sujetos seleccionados de 20 o más años, entre julio de 2014 y enero de 2017.

El tamaño mínimo de la muestra se calculó utilizando la fórmula de muestreo aleatorio para grandes poblaciones, considerando una muestra suficiente para detectar la condición menos prevalente en Venezuela (diabetes 7,7\%) (5). Se estableció un nivel de confianza del $95 \%$, un error máximo admisible de $1,55 \%$ y una tasa de respuesta de $70 \%$ para un mínimo de 525 sujetos en la región de los Andes. Se evaluaron 5 poblaciones: en el Estado Mérida durante noviembre de 2015 a marzo de 2016: La Venta (rural - Páramo), el Llano y Belén (urbano - centro de la ciudad de Mérida) y Tovar (urbano); en el Estado Trujillo, la Mesa de Esnujaque (urbano). La tasa de respuesta fue $76,6 \%$. Se incluyeron todos los sujetos mayores de 20 años que se encontraban en las casas seleccionadas y firmaron el consentimiento informado. Se excluyeron las mujeres embarazadas y sujetos con incapacidad para mantenerse de pie o comunicarse verbalmente. El protocolo de estudio fue diseñado acorde con la declaración de Helsinki y aprobado por el Comité Nacional de Bioética (CENABI) de Venezuela. El presente reporte está presentado acorde a las recomendaciones STROBE (The Strengthening the Reporting of Observational Studies in Epidemiology) (10).

\section{Datos Clínicos y Antropométricos}

Durante la visita de reclutamiento a las casas seleccionadas se realizó la invitación a los participantes a una evaluación en un centro de campo, se informó sobre el estudio, se entregó y explicó el consentimiento informa- 
do, se tomaron datos de identificación, uso de servicios de salud, nivel académico y el cuestionario de estrato social (11). Todos los sujetos fueron evaluados en un centro de campo de la comunidad por personal entrenado acorde a un protocolo estándar. Durante la evaluación se completaron los datos generales y cuestionarios: psicológico, actividad física, insuficiencia cardiaca, frecuencia de alimentos y adherencia a la dieta mediterránea. La talla fue medida usando un estadiómetro portátil (Seca 206®) seca gmbh \& co. Hamburg / Germany). El peso de cada individuo, vestido con vestimenta ligera y sin zapatos, fue medido con una escala calibrada (Tanita UM-081 @ Japan). El índice de masa corporal fue calculado como $\mathrm{IMC}=\mathrm{Kg}$ de peso $/ \mathrm{m}^{2}$ de talla.

\section{Definición de Variables}

Se aplicaron las definiciones de sobrepeso y obesidad de la Organización Mundial de la salud (OMS): bajo peso = IMC $<18,5 \mathrm{~kg} /$ $\mathrm{m}^{2}$; peso normal $=$ IMC 18 a $24,9 \mathrm{~kg} / \mathrm{m}^{2} ;$ sobrepeso = IMC 25 a $29,9 \mathrm{~kg} / \mathrm{m}^{2}$; obesidad $=\mathrm{IMC} \geq 30 \mathrm{~kg} / \mathrm{m}^{2}$. La obesidad abdominal se estableció de acuerdo con los límites propuestos para América Latina, circunferencia abdominal $\geq 94 \mathrm{~cm}$ en hombres $\mathrm{y} \geq 90 \mathrm{~cm}$ en mujeres (12). Alto porcentaje de grasa corporal se definió según la OMS: $>25 \%$ en hombres y $>35 \%$ en mujeres (13).

\section{Análisis de Datos}

Todos los datos fueron analizados usando el programa SPSS 20 (IBM corp. Released 2011. Armonk, NY: USA). Todas las variables continuas fueron inicialmente analizadas con el test de normalidad de Kolmogorov-Smirnov. Las variables fueron presentadas como medias \pm error estándar de la media y sus diferencias fueron medidas con la prueba $\mathrm{t}$ de Student $\mathrm{y}$ ANOVA para más de dos poblaciones. Las proporciones fueron mostradas como porcentaje. El test de Chi-cuadrado fue utilizado para establecer la diferencia de las variables categóricas. Un valor de $\mathrm{p}<0,05$ fue considerado estadísticamente significativo.

\section{RESULTADOS}

Un tercio de la población fueron mujeres. El promedio de edad fue de 51,1 $\pm 0,80$ años, mayor en hombres que las mujeres $(p<0,05)$ (Tabla I). Los hombres presentaron mayor talla y peso que las mujeres, pero similar IMC.

\section{Características de los sujetos por poblaciones evaluadas}

Los sujetos más jóvenes se encontraron en la población de la Venta, y los de mayor estatura en las poblaciones de El Llano y To$\operatorname{var}(\mathrm{p}<0,05)$ (Tabla II).

TABLA I

CARACTERÍSTICAS ANTROPOMÉTRICAS DE LOS SUJETOS SEGÚN GÉNERO, DE LA REGIÓN DE LOS ANDES, VENEZUELA.

\begin{tabular}{lccc}
\hline & Hombres & Mujeres & Total \\
\hline $\mathrm{n}(\%)$ & $142(34,0)$ & $276(66,0)$ & $418(100,0)$ \\
Edad (años)* & $53,6 \pm 1,43$ & $49,8 \pm 0,96$ & $51,1 \pm 0,80$ \\
Talla $(\mathrm{m}) *$ & $1,66 \pm 0,00$ & $1,55 \pm 0,00$ & $1,59 \pm 0,00$ \\
Peso $(\mathrm{kg}) *$ & $74,0 \pm 1,16$ & $64,5 \pm 0,81$ & $67,7 \pm 0,70$ \\
IMC $\left(\mathrm{kg} / \mathrm{m}^{2}\right)$ & $26,6 \pm 0,35$ & $26,7 \pm 0,31$ & $26,7 \pm 0,24$ \\
CA $(\mathrm{cm})$ & $94,6 \pm 0,95$ & $93,9 \pm 0,74$ & $93,9 \pm 0,59$ \\
Grasa corporal $(\%)$ & $25,6 \pm 7,08$ & $34,4 \pm 0,46$ & $31,6 \pm 0,42$ \\
\hline
\end{tabular}

Los datos continuos están representados como medias \pm error estándar de la media. * $\mathrm{p}<0,05$.

Abreviaturas: CA: circunferencia abdominal. IMC: índice de masa corporal.

Vol. 59(3): $242-249,2018$ 


\section{TABLA II}

CARACTERÍSTICAS ANTROPOMÉTRICAS DE LOS SUJETOS SEGÚN LA POBLACION EVALUADA, EN LA REGIÓN DE LOS ANDES, VENEZUELA.

\begin{tabular}{lccccc}
\hline Poblaciones & La Venta & Belén & El Llano & Tovar & La Mesa \\
\hline n (\%) & $73(17,5)$ & $116(27,8)$ & $71(17,0)$ & $34(8,1)$ & $124(29,6)$ \\
Edad (años)* & $47,2 \pm 1,81$ & $54,2 \pm 1,54$ & $52,4 \pm 1,88$ & $52,7 \pm 2,87$ & $49,3 \pm 1,51$ \\
Talla (m)* & $1,57 \pm 0,01$ & $1,58 \pm 0,00$ & $1,61 \pm 0,01$ & $1,61 \pm 0,01$ & $1,54 \pm 0,00$ \\
Peso (kg) & $66,4 \pm 1,46$ & $68,2 \pm 1,51$ & $70,4 \pm 1,66$ & $66,8 \pm 2,23$ & $64,1 \pm 1,50$ \\
IMC (kg/m $\left.{ }^{2}\right)$ & $26,8 \pm 0,51$ & $27,1 \pm 0,53$ & $26,8 \pm 0,57$ & $25,7 \pm 0,84$ & $26,6 \pm 0,56$ \\
CA (cm) & $94,0 \pm 1,30$ & $95,3 \pm 1,29$ & $94,2 \pm 1,34$ & $89,7 \pm 2,16$ & $93,4 \pm 0,97$ \\
Grasa corporal (\%) & $30,8 \pm 0,99$ & $33,4 \pm 0,80$ & $31,6 \pm 1,03$ & $29,9 \pm 1,58$ & $30,7 \pm 0,73$ \\
\hline
\end{tabular}

Los datos continuos están representados como medias \pm error estándar de la media. * $\mathrm{p}<0,05$.

Abreviaturas: CA: circunferencia abdominal. IMC: índice de masa corporal.

Prevalencia de obesidad por género

Alrededor del $65 \%$ de los sujetos evaluados presentó alteración del estado nutricional. La prevalencia de obesidad fue de $22,2 \%$, mucho mayor en mujeres que en hombres $(25,5 \%$ y $15,7 \%$, respectivamente, $\mathrm{p}$ $=0,014$ ) (Tabla III). La prevalencia de alto porcentaje de grasa y obesidad abdominal, las cuales afectaron a cerca de la mitad de la muestra, fue similar entre géneros. El bajo peso fue más elevado en las mujeres en comparación con los hombres $(\mathrm{p}=0,014)$.

Prevalencia de obesidad por poblaciones

La población de Tovar mostró un estado nutricional distinto al resto de las loca- lidades evaluadas. Con una prevalencia de bajo peso 5 veces mayor que el promedio del resto de las poblaciones $(\mathrm{p}<0,05)$ y las más baja prevalencia de obesidad abdominal ( $p$ = no significativa), sin embargoo, el aumento de grasa corporal fue discretamente similar (Tabla IV).

\section{DISGUSIÓN}

El 22,2\% de la población evaluada en la región de los Andes, presentó obesidad definida según el IMC, y cerca de la mitad, obesidad abdominal o alto porcentaje de grasa. Según el IMC, la obesidad fue 1,5 veces mayor y el bajo peso 4 veces mayor en muje-

TABLA III

PREVALENCIA DE OBESIDAD ENTRE GÉNEROS SEGÚN LA POBLACIÓN EVALUADA EN LA REGIÓN DE LOS ANDES, VENEZUELA

\begin{tabular}{lccc}
\hline & Hombres & Mujeres & Total \\
\hline Bajo peso (\%)" & 0,7 & 3,3 & 2,4 \\
Normopeso (\%) & 35,0 & 35,8 & 35,5 \\
Sobrepeso (\%)" & 48,6 & 35,4 & 39,9 \\
Obesidad (\%)" & 15,7 & 25,5 & 22,2 \\
Obesidad Abdominal (\%) & 45,9 & 55,7 & 50,8 \\
Alto \% grasa & 49,1 & 47,4 & 48,2 \\
\hline "p $<0,014$. & & &
\end{tabular}




\section{TABLA IV}

PREVALENCIA DE OBESIDAD EN LAS POBLACIONES EVALUADAS

DE LA REGIÓN DE LOS ANDES, VENEZUELA.

\begin{tabular}{lccccc}
\hline Poblaciones & La Venta & Belén & El Llano & Tovar & La Mesa \\
\hline Bajo peso (\%)* & 1,4 & 2,6 & 2,8 & 8,8 & 0,8 \\
Normopeso (\%) & 32,9 & 36,0 & 32,4 & 32,4 & 39,0 \\
Sobrepeso (\%) & 42,5 & 39,5 & 40,8 & 44,1 & 36,6 \\
Obesidad (\%) & 23,3 & 21,9 & 23,9 & 14,7 & 23,6 \\
Obesidad Abdominal (\%) & 57,5 & 65,5 & 59,2 & 38,2 & $\mathbf{5 8 , 5}$ \\
Alto \% grasa & 49,3 & 58,9 & $\mathbf{5 8 , 6}$ & 47,1 & $\mathbf{5 1 , 2}$ \\
\hline
\end{tabular}

Las tasas como porcentaje ( $95 \%$ intervalo de confianza). *Bajo peso de individuos de Tovar comparado con el promedio de las otras poblaciones, $\mathrm{p}<0,05$.

res que en hombres. La población de Tovar presentó la menor prevalencia de obesidad y mayor prevalencia de bajo peso. Tovar fue la última población evaluada en la región de los Andes (marzo 2016), momento en que había comenzado la severa escasez de alimentos en el país lo que pudiera justificar esta diferencia.

La prevalencia de obesidad encontrada en este estudio fue mayor a la prevalencia mundial $12,8 \%$ (4), pero menor a la prevalencia de América Latina 27\% (4). El estudio VEMSOLS, realizado en 3 regiones de Venezuela, entre los años 2006 a 2010, reportó una prevalencia de obesidad de $24,3 \%$ en la población urbana de los Andes (7), mayor a lo reportado en este estudio y rural $12,1 \%$ (7), la mitad a lo observado en este estudio. La prevalencia de bajo peso reportada en el estudio VEMSOLS fue 1,1\%, menos de la mitad a lo observado en el presente estudio. El diferente contexto sociopolítico en el que se desarrollaron ambos estudios pudiera justificar sus diferencias. Cuando se tomaron los datos del EVESCAM, había comenzado una situación en el país, de incremento de precios de los alimentos, asociado a escasez de múltiples productos alimentarios, lo que sin duda ha ido modificando el patrón alimentario del Venezolano (14). Igualmente, el sistema de transporte público comenzaba a mermar, lo que pudiera incrementar el ni- vel de actividad física de los individuos, que sumado al cambio del patrón alimentario, pudiera explicar una menor prevalencia de obesidad y mayor prevalencia de bajo peso. Sin embargo, son necesarios otro tipo de estudios para determinar esta hipótesis.

Es evidente como la prevalencia de obesidad definida por el IMC fue más elevada que la prevalencia mundial, que abarca las regiones de África y la India, pero notoriamente menor a lo reportado para la región y para las mismas localidades con respecto a estudios previos. En contraparte al descenso de obesidad en la zona urbana, el bajo peso aumentó en la población evaluada.

La nueva perspectiva de obesidad como enfermedad crónica y compleja, impone un nuevo enfoque clínico y epidemiológico en los pacientes con acumulación, distribución y función anómala de la masa grasa. La definición actual de obesidad se basa en el IMC (15). La Asociación Americana de Endocrinólogos Clínicos (AACE) y el Colegiio Americano de Endocrinología (ACE) en 2014 plantearon que el IMC solo, no refleja el impacto del aumento de peso en la salud o el bienestar del individuo (16), en consecuencia, propusieron una definición médica de la obesidad, que incorpora no sólo el uso del IMC, sino también otras evaluaciones físicas que incluyen la presencia y gravedad de las complicaciones relacionadas con la obe- 
sidad, para reflejar el impacto de la adiposidad en la salud de pacientes individuales (AACE/ACE Marco Conceptual) $(16,17)$. Si se usan los datos del VEMSOLS y el mareo conceptual propuesto por la AACE/ACE, la prevalencia de obesidad para Venezuela en 2010 fue de $63,1 \%$ (7).

El presente estudio no planteó como objetivo determinar la prevalencia de obesidad acorde a este marco conceptual, pero observamos como el número de sujetos con sobrepeso más obesidad $(60,2 \%)$ es similar a lo observado en el VEMSOLS. Es decir, a pesar de la aparente reducción de la obesidad en la población de los Andes en las zonas urbanas, se observa que se mantiene una elevada proporción de sujetos con peso elevado (sobrepeso + obesidad, u obesidad abdominal o alto porcentaje de grasa), lo cual, está asociado en el 99\% de los casos de la población Venezolana a factores de riesgo relacionados con la obesidad (7). Adicionalmente, se sumó el incremento del bajo peso, el cual condiciona otro tipo de factores de riesgo para morbimortilidad. La población de los Andes puede estar más delgada pero no necesariamente más saludable (7).

Dentro de las limitaciones de este estudio se encuentra que el tamaño de la muestra estimada para la región, no permitió el análisis de los factores de riesgo asociados a obesidad, sobrepeso y bajo peso. Esta limitación será cubierta con el análisis de los datos nacionales. Dentro de las fortalezas encontramos la representatividad de la muestra y el tipo de muestreo realizado permite la extrapolación de los resultados al resto de la población.

En conclusión, basados en el IMC, se encontró una baja prevalencia de obesidad y alta prevalencia de bajo peso en la región de los Andes venezolanos, siendo la obesidad y bajo peso mayor en mujeres que en hombres. Así mismo, entre las poblaciones evaluadas, la población de Tovar mostró una alta prevalencia de bajo peso y menor prevalencia de obesidad en comparación al resto de las poblaciones estudiadas. Los ele- mentos que conducen a estas prevalencias deben ser investigados.

\section{AGRADEGIMIENTOS}

A la Sociedad Venezolana de Medicina Interna (SVMI) y todos sus miembros, y a los múltiples colaboradores en la recolección de datos, quienes permitieron la realización de este estudio. Así como a los pacientes que decidieron participar en el mismo. Este estudio fue financiado parcialmente por un Grant aportado por Laboratorios Novartis (Caracas, Venezuela) y el apoyo de financiero particulares.

\section{REFERENCIAS}

1. GBD 2015 Risk Factors Collaborators. Global, regional, and national comparative risk assessment of 79 behavioural, environmental and occupational, and metabolic risks or clusters of risks, 1990-2015: a systematic analysis for the Global Burden of Disease Study 2015. Lancet. 2016; 388(10053):1659-1724.

2. Jensen MD, Ryan DH, Apovian CM, Ard JD, Comuzzie AG, Donato KA, Hu FB, Hubbard VS, Jakicic JM, Kushner RF, Loria CM, Millen BE, Nonas CA, Pi-Sunyer FX, Stevens J, Stevens VJ, Wadden TA, Wolfe BM, Yanovski SZ. 2013 AHA/ACC/TOS guideline for the management of overweight and obesity in adults: a report of the American College of Cardiology/American Heart Association Task Foree on Practice Guidelines and The Obesity Society. J Am Coll Cardiol 2014;63(25 Pt B):2985-3023.

3. Zhao G, Ford ES, Dhingra S, Li C, Strine TW, Mokdad AH. Depression and anxiety among US adults: associations with body mass index. Int J Obes (Lond) 2009; 33(2):257-266.

4. NCD Risk Factor Collaboration (NCDRisC). Trends in adult body-mass index in 200 countries from 1975 to 2014: a pooled analysis of 1698 population-based measurement studies with 19.2 million participants. Lancet 2016; 387 (10026):1377-1396.

5. Nieto-Martínez R, Hamdy $O$, Marante D, Marulanda M, Marchetti A, Hegazi R, Me- 
chanick J. Transcultural diabetes nutrition algorithm (tDNA): Venezuelan application. Nutrients 2014;6(4):1333-1363.

6. González-Rivas JP, Nieto-Martínez RE, Molina de González Méndez T, García RJ, Ugel E, Osuna D, LS. Prevalencia de síndrome metabólico, obesidad y alteración de la ǵlucemia en ayunas en adultos del páramo del Estado Mérida, Venezuela (estudio VEMSOLS). Med Interna (Caracas) 2012;28(1):262-267.

7. Nieto-Martínez R, González-Rivas J, Ugel E, Brajkovich I, Risquez A, Garvey WT, Mechanick JI. Application of the AACE/ ACE advanced framework for the diagnosis of obesity and cardiometabolic disease staging in a general population from three regions of Venezuela: The VEMSOLS study results. Endocr Pract 2018;24(1):6-13.

8. Nieto-Martínez R, Marulanda MI, Ugel E, Duran M, González-Rivas J, Patiño M, López-Gómez L, Monsalve P, Marcano H, Barengo N, Aschner P, Flórez H. Venezuelan Study of Cardio-metabolic Health (EVESCAM): General description and sampling. Med Interna 2015;31(2):102-111.

9. Nieto-Martínez R, Marulanda MI, González-Rivas JP, Ugel E, Durán M, Barengo N, Aschner P, Patiño M, LL-G, Monsalve P, Mareano H, Florez H. CardioMetabolic Health Venezuelan Study (EVESCAM): Design and Implementation. Invest Clin 2017;58(1):56-61.

10. von Elm E, Altman DG, Egger M, Pocock SJ, Gotzsche PC, Vandenbroucke JP. The Strengthening the Reporting of Observational Studies in Epidemiology (STROBE) statement: guidelines for reporting observational studies. PLoS Med 2007;4(10):e296.

11. Méndez-Castellano H, Méndez MC. Estratificación social y humana. Método de Graffar modificado. Arch Venez Puer Pediatr 1986;49:93-104.
12. Aschner P, Buendia R, Brajkovich I, Gonzalez A, Figueredo R, Juarez XE, Uriza F, Gomez AM, Ponte CI. Determination of the cutoff point for waist circumference that establishes the presence of abdominal obesity in Latin American men and women. Diabetes Res Clin Pract 2011;93(2):243-247.

13. AACE/ACE Obesity Task Foree. AACE/ ACE position statement on the prevention, diagnosis, and treatment of obesity. Endocr Pract 1998;4:297-350.

14. ACAP. Humanitarian Overview: an analysis of key crises into 2018. Accessed on January 29,2018 . Available online: https://www. acaps.org/special-report/humanitarianoverview-analysis-key-crises-2018.

15. Clinical Guidelines on the Identification, Evaluation, and Treatment of Overweight and Obesity in Adults -The Evidence Report. National Institutes of Health. Obes Res 1998;6 Suppl 2:51S-209S.

16. Garvey WT, Garber AJ, Mechanick JI, Bray GA, Dagogo-Jack S, Einhorn D, Grunberger G, Handelsman Y, Hennekens CH, Hurley DL, McGill J, Palumbo P, Umpierrez G, On Behalf Of The Aace Obesity Scientific Committee. American association of clinical endocrinologists and american college of endocrinology position statement on the 2014 advanced framework for a new diagnosis of obesity as a chronic disease. Endocr Pract 2014;20(9):977-989.

17. Garvey WT, Mechanick JI, Brett EM, Garber AJ, Hurley DL, Jastreboff AM, Nadolsky K, Pessah-Pollack R, Plodkowski R. American Association Of Clinical Endocrinologists And American College Of Endocrinology Comprehensive Clinical Practice Guidelines For Medical Care Of Patients With Obesity. Endocr Pract 2016;22 Suppl 3:1-203. 